\section{Osteochondrosis dissecans am sagittalen Rollkamm von Mc III und Mt III beim Pferd}

\author{
J. V. Yovich, C. W. McIlwraith und T. S. Stashak \\ College of Veterinary Medicine, Colorado State \\ University, Fort Collins
}

\section{Einführung}

Beim Pferd treten die Manifestationsformen der Osteochondrosis dissecans am häufigsten in den Sprung-, Knieund Schultergelenken auf. Von solchen Fällen gibt es viele Beschreibungen (siehe alle Literaturangaben). Die Osteochondrosis dissecans am Sagittalkamm der dritten Metakarpal- und Metatarsalknochen ist schriftlich jedoch nur kurz erwähnt (McIlwraith, 1982; Fischer et al., 1984). Wir gehen im vorliegenden Bericht auf diese Lokalisationsform bei 8 Pferden ein, die an der Colorado State University innerhalb von 18 Monaten zur Untersuchung vorgestellt wurden.

\section{Fall 1}

Ein 11 Monate altes Araberhengstfohlen kam wegen vermehrter Füllung der beiden hinteren Fesselgelenke und zwecks Beurteilung einer Knochenexostose am Os metacarpale tertium der linken Vordergliedmaße zur Untersuchung. Im Trab ging das Fohlen nicht lahm, und die Knochenexostose schien nicht problematisch zu sein. Durch passives Beugen ließ sich an allen Fesselgelenken eine Schmerzreaktion auslösen. Die Röntgenuntersuchung des linken Vorderbeins bestätigte die Knochenexostose und ergab, daß Knochenveränderungen im Metakarpophalangealgelenk vorhanden waren. Am dorsoproximalen Rand des Sagittalkamms, zwischen den distalen Kondylen des dritten Metakarpalknochens, waren Bereiche mit vermehrt dichtem Knochengewebe sichtbar. Daran angrenzend wies der subchondrale Knochen des Sagittalkamms Unregelmäßigkeiten auf. Die Röntgenaufnahmen der 3 anderen Fesselgelenke zeigten in beiden Metatarsophalangealgelenken ähnliche Veränderungen am Knochen wie diejenigen im linken vorderen Fesselgelenk. Das rechte Metakarpophalangealgelenk war offenbar, was die Knochenstruktur betraf; ohne Befund. Im hinteren linken Fesselgelenk war eine Stelle mit verdichtetem Knochengewebe in der Nähe des dorsoproximalen Rands des Sagittalkamms des Os metatarsale tertium zu erkennen. Unregelmäßigkeiten des subchondralen Knochens und sklerotische Veränderungen ließen sich im Sagittalkamm in dem die Knochenverdichtungsstellen umgebenden Bereich ausmachen. Im rechten hinteren Fesselge-

\begin{abstract}
Zusammenfassung
In einem Zeitraum von 18 Monaten lautete bei 8 Pferden die Diagnose auf Osteochondrosis dissecans am Sagittalkamm der dritten Metakarpal- und Metatarsalknochen. 7 Pferde waren dabei 21/2 Jahre alt oder jünger. Die typischen Befunde bestanden in vermehrter Füllung der erkrankten Fesselgelenke und einer Schmerzreaktion bei der Beugung des Fesselgelenks. Im Trab stand die Lahmheit einer einzelnen Gliedmaße im Vordergrund. Mit der Fesselgelenksbeugeprobe ließ sich jedoch oft auch eine Lahmheit anderer betroffener Gliedmaßen hervorrufen. Bei allen Pferden waren mittels Röntgenuntersuchung Knochenläsionen (Abflachung, Erosionen oder Absplitterung) am Sagittalkamm mindestens zweier Fesselgelenke festzustellen. Ein Zusammenhang zwischen dem Einsetzen klinischer Erscheinungen und der Schwere der röntgenologischen Veränderungen bestand nicht in allen Fällen.
\end{abstract}

lenk glichen 2 Punkte mit vermehrt dichtem Knochengewebe beim Sagittalkamm den Veränderungen im linken Metakarpophalangeal- und linken Metatarsophalangealgelenk (Abb. 1). Die Empfehlung lautete auf Schonung mit Weidegang und Nachuntersuchung in 3 Monaten.

Bei der Untersuchung im Alter von 14 Monaten war im Trab keine deutliche Lahmheit vorhanden. Alle Fesselgelenke waren jedoch vermehrt gefüllt und beim Abbeugen in der Bewegung eingeschränkt. Durch passive Beugung ließ sich in allen Fesselgelenken eine Schmerzreaktion hervorrufen. Nach der Beugeprobe am Fesselgelenk jeder Gliedmaße ging das Pferd im Trab lahm. Die Röntgenuntersuchung aller Fesselgelenke erbrachte, daß die Veränderungen am Knochen seit der Erstuntersuchung nicht fortgeschritten waren. Man empfahl weiterhin Schonung mit Weidegang. Die Nachuntersuchung im Alter von 26 Monaten ergab keine sichtbare Lahmheit. Die Fesselgelenke waren weniger stark gefüllt. Die Röntgenbefunde lauteten für alle Fesselgelenke auf dieselben Veränderungen, ausgenommen eine relativ geringe periartikuläre Osteophytenbildung. Während des darauffolgenden Jahres wurde das junge Pferd unter dem Sattel und an der Longe gearbeitet. Die Untersuchung im Alter von 38 Monaten ließ eine leicht vermehrte Füllung aller Fesselgelenke erkennen. Das Pferd ging im Trab nicht lahm. Die Beugeprobe am linken Metakarpophalangealgelenk löste aber eine Schmerzreaktion und eine geringgradige vorübergehende Lahmheit in diesem Bein im Trab aus. Durch Beugung der übrigen Fesselgelenke war kein Schmerz.hervorzurufen. Die Röntgenaufnahmen aller Fesselgelenke erbrachten keine veränderten Befunde gegenüber denjenigen der Röntgenuntersuchung des Pferdes mit 26 Monaten. Lediglich die Knochenverdichtungen in der Nähe des Sagittalkamms des rechten Os metatarsale tertium hatten an Größe zugenommen.

\section{Fall 2}

Eine 2jährige Quarter-Horse-Stute kam wegen vermehrter Füllung beider Metakarpophalangealgelenke zur Untersuchung. Nach dem Rennen war eine Lahmheit aufgetreten. Die Stute ging im Trab vorn links lahm, und die Beugung der Fesselgelenke war vorn beidseits schmerzhaft. Die 


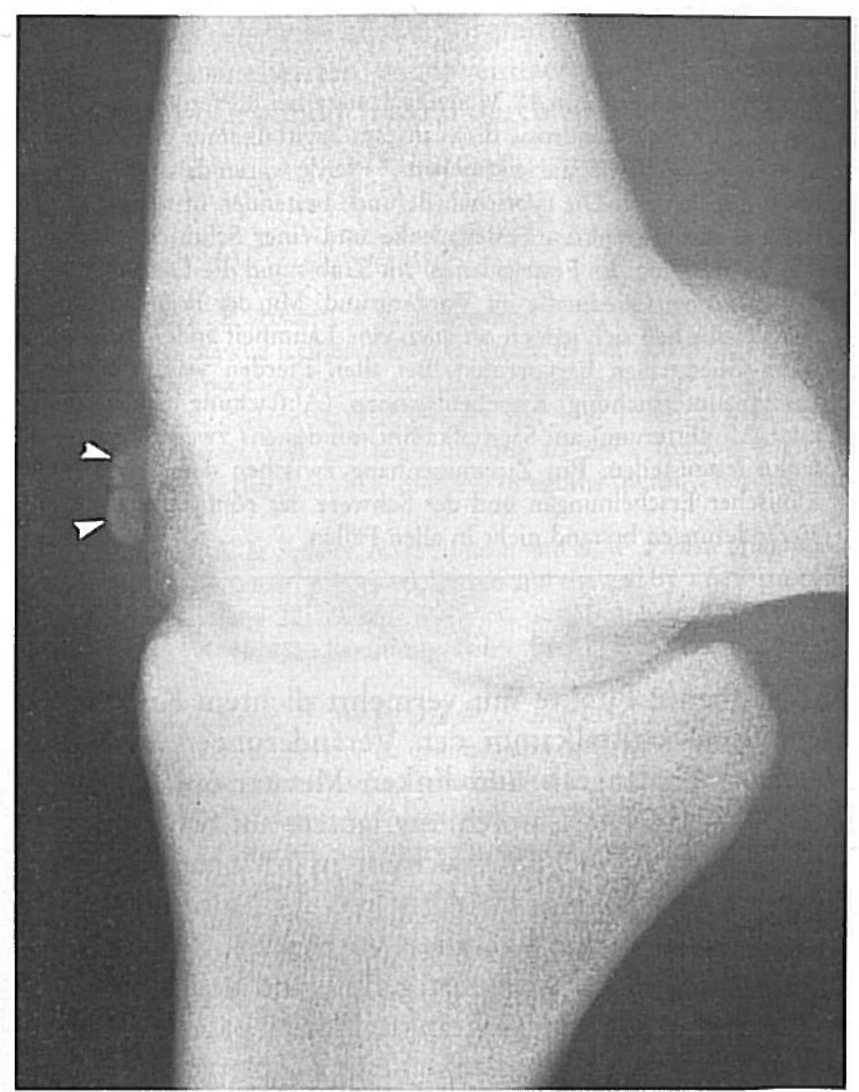

Abb. 1: Seitliche Röntgenaufnahme vom rechten Metatarsophalangealgelenk in Schrägprojektion, Pferd 1. Zu beachten die Bereiche mit verdichtetem Knochengewebe (weiße Pfeile), angrenzend an den Sagittalkamm.

Röntgenaufnahmen beider Metakarpophalangealgelenke zeigten eine Abflachung des subchondralen Knochens am Sagittalkamm beider Ossa metacarpalia tertia. Die Stute erhielt 3 Monate Schonung mit Weidegang und wurde dann nachuntersucht. Die röntgenologischen Befunde dieser Untersuchung waren dieselben wie zuvor. Es war lediglich eine leichte Osteophytenbildung an der dorsoproximalen Fläche der Phalanx proximalis in den beiden vorderen Fesselgelenken sichtbar.

Nach 6monatiger Schonfrist waren bei der Stute keine vermehrte Gelenkfüllung und keine weiteren röntgenologischen Veränderungen festzustellen. Sie nahm das Training auf und ging 7 Rennen, von welchen sie eines gewann, ohne Anzeichen von Lahmheit.

7 Monate, nachdem die Stute an ihrem letzten Rennen teilgenommen hatte, machte man Röntgenaufnahmen von beiden Metakarpophalangealgelenken. Darauf traten eine Abflachung und leichte Unregelmäßigkeiten der Dorsalfläche des Sagittalkamms beider Ossa metacarpalia tertia in Erscheinung. Hinweise auf ein Fortschreiten der Gelenkserkrankung fanden sich am Knochen jedoch nicht.

\section{Fall 3}

Der Vorbericht einer 12 Monate alten Quarter-Horse-Stute (einer Halbschwester der Stute des Fall 2) beschrieb eine seit 21/2 Monaten bestehende Lahmheit in allen Gliedma-

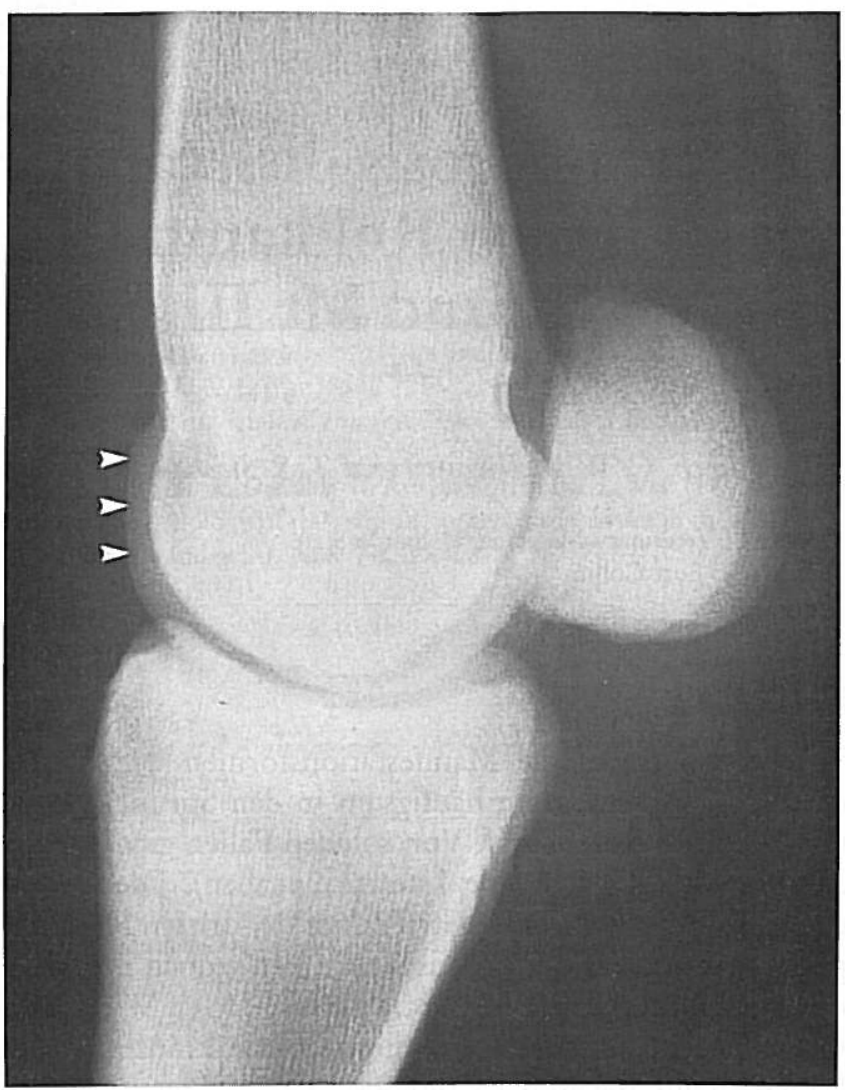

Abb. 2: Seitliche Röntgenaufnahme vom rechten Metakarpophalangealgelenk, Pferd 3 . Zu beachten die Abflachung an der Dorsalfläche des Sagittalkamms (weiße Pfeile).

ßen. Beide Metakarpophalangealgelenke waren vermehrt gefüllt, und am Fesselgelenk der rechten Hintergliedmaße war eine Umfangsvermehrung von fester Konsistenz vorhanden. Die passive Beugung rief an jedem dieser Gelenke eine Schmerzreaktion hervor. Die Röntgenaufnahmen zeigten eine Abflachung der Dorsalfläche des Sagittalkamms beider Ossa metacarpalia tertia (Abb. 2). An der distalen Fläche der proximalen Phalanx und an der dorsoproximalen Fläche der Phalanx media des rechten Hinterbeins war eine Periostreaktion sichtbar. Die Stute erhielt $3 \mathrm{Mo}$ nate Boxenruhe und anschließend 9 Monate Auslauf auf der Weide. Die Untersuchung im Alter von 21 Monaten hatte keine Lahmheit und keine vermehrte Füllung der Fesselgelenke zum Ergebnis. Auf den Röntgenaufnahmen beider vorderer Fesselgelenke war eine verminderte Abflachung des Sagittalkamms der dritten Metakarpalknochen, verglichen mit dem Grad der Erscheinungen im Alter von 12 Monaten, festzustellen.

\section{Fall 4}

Die Überweisung einer 10 Monate alten American-Saddlebred-Stute erfolgte mit dem Vorbericht, sie leide seit ihrer Geburt an einer Atemwegserkrankung sowie an wechselnden Gelenkschwellungen und Lahmheit. Alle Fesselgelenke waren vermehrt gefüllt, die Stute zeigte einen steifen Gang und bewegte sich nur widerwillig. Die Röntgenun- 
tersuchung der Fesselgelenke ergab, daß der subchondrale Knochen Unregelmäßigkeiten aufwies und von der Dorsalfläche des Sagittalkamms aller Ossa metacarpalia und metatarsalia tertia kleine Knochenfragmente abgelöst waren (Abb. 3). Auf den Röntgenaufnahmen beider Sprung- und Kniegelenke waren keine Veränderungen sichtbar. Man euthanasierte die Stute wegen ihrer schweren Bronchopneumonie und einer Nierenerkrankung.

Die makroskopische pathologische Untersuchung der Fesselgelenke zeigte Läsionen an allen Ossa metacarpalia und metatarsalia tertia, die auf dem Sagittalkamm lokalisiert waren. An der Dorsalfläche des Sagittalkamms fanden sich Knorpelerosionen und Unregelmäßigkeiten des subchondralen Knochens. Kleine osteochondrale Fragmente in den Defekten besaßen bindegewebige Verbindung zum subchondralen Knochen.

\section{Fall 5}

Eine 26 Monate alte Araberstute kam auf Überweisung zur kosmetischen Revision einer alten Rißwunde in die Klinik. In den vergangenen 5 Monaten war die Stute regelmäßig an der Longe gearbeitet worden. Es fiel eine vermehrte Füllung aller Fesselgelenke ins Auge. Die Stute ging lahm, im Trab schonte sie das linke Vorderbein. Die Beugung der beiden Metakarpophalangealgelenke sowie des rechten Metatarsophalangealgelenks war schmerzhaft. Die Röntgenaufnahmen der Metakarpophalangealgelenke ließen eine ausgedehnte Aussparung im Knochen der Dorsalfläche des Sagittalkamms beider Ossa metacarpalia tertia erkennen (Abb. 4). In dem betroffenen Bereich des Sagittalkamms war die Oberfläche unregelmäßig. Bei der Röntgenuntersuchung des rechten Metatarsophalangealgelenks waren keine Knochenveränderungen nachzuweisen.

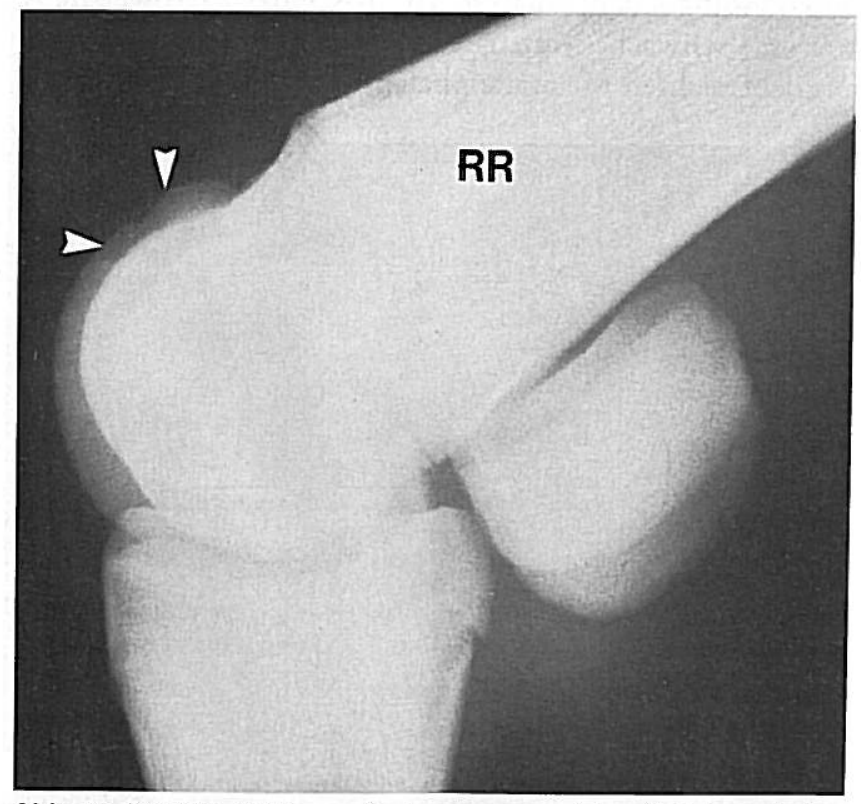

Abb. 3: Seitliche Röntgenaufnahme vom rechten Metatarsophalan. gealgelenk, Pferd 4. Zu beachten die Erosionen und Unregelmäßigkeiten auf der Dorsalfiäche des Sagittalkamms (zwischen den weißen Pfeilen).

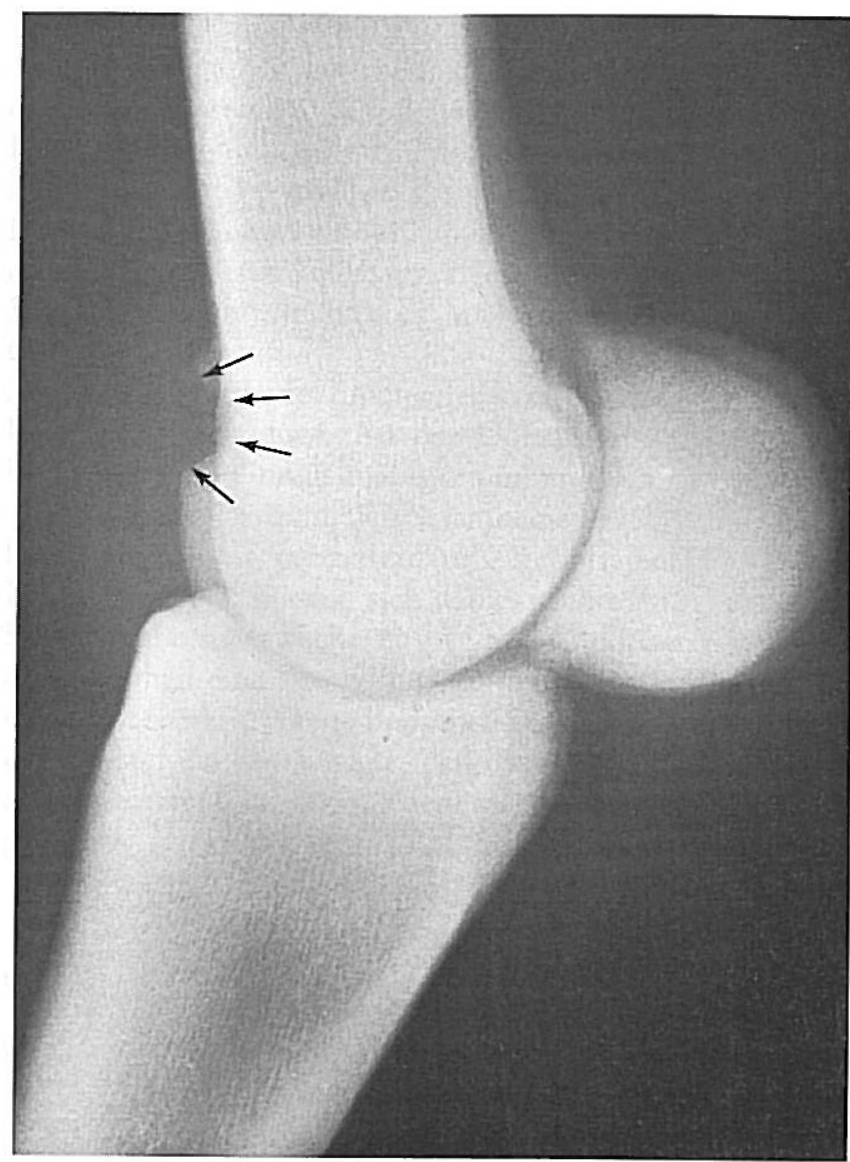

Abb. 4: Seitliche Röntgenaufnahme vom linken Metakarpophalangealgelenk, Pferd 5. Zu beachten der Knochendefekt am Sagittalkamm (konvergierende schwarze Pfeile bezeichnen den Umriß).

Die Stute erhielt 6 Monate Schonung mit Weidegang. Danach war die vermehrte Füllung der Gelenke zurückgegangen und keine Lahmheit mehr sichtbar. Sie nahm aber das Training nicht wieder auf.

\section{Fall 6}

Ein 6 Monate altes Quarter-Horse-Hengstfohlen wurde wegen einer 10 Tage anhaltenden Durchfallerkrankung stationär behandelt. Das Fohlen hatte einen steifen Gang, und die Intensität der Lahmheit in den einzelnen Gliedmaßen wechselte während seines Klinikaufenthalts täglich. Alle Fesselgelenke waren geschwollen. Die Röntgenaufnahmen der Fesselgelenke zeigten Stellen von verdichtetem Knochengewebe, angrenzend an Unregelmäßigkeiten des subchondralen Knochens am Sagittalkamm des rechten Os metacarpale tertium und beider Ossa metatarsalia tertia (Abb. 5 und 6).

Auf den Röntgenaufnahmen des linken vorderen Fesselgelenks sowie beider Sprung- und Kniegelenke waren keine Knochenveränderungen zu sehen. Das Hengstfohlen wurde auf Wunsch der Besitzer wegen der hochgradigen persistierenden Lahmheit euthanasiert.

Die Sektion erbrachte eine vergrößerte Menge Synovialflüssigkeit in den erkrankten Gelenken mit hervortretender Proliferation der Synovialis-Villi. An der Dorsalfläche 


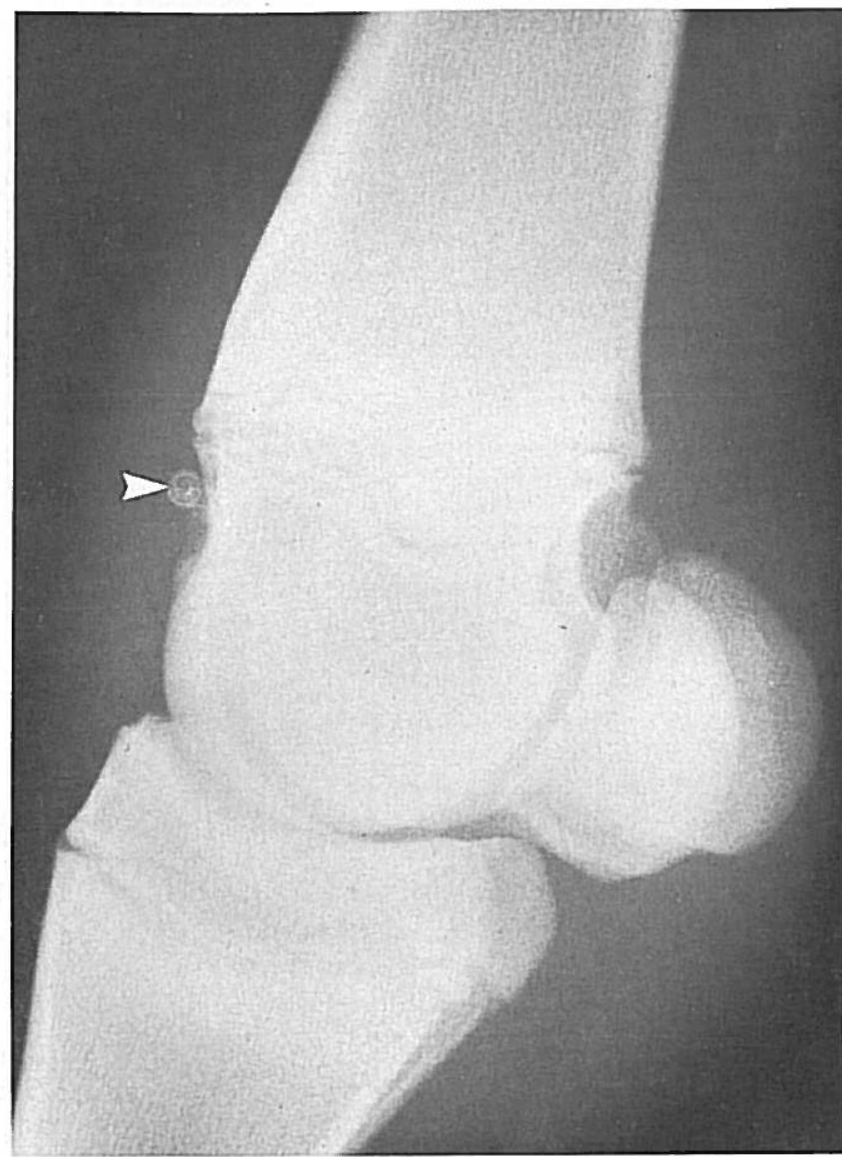

Abb. 5: Seitliche Röntgenaufnahme vom linken Metatarsophalangealgelenk, Pferd 6. Zu beachten die verdichtete Knochengewebsstelle (weißer Pfeil), angrenzend an einen Bereich von Unregelmäßigkeiten am Sagittalkamm.

des Sagittalkamms des rechten Os metacarpale tertium und beider Ossa metatarsalia tertia hatten sich Knorpelerosionen und Knorpelflaps gebildet, und der subchondrale Knochen war freigelegt (Abb. 7).

\section{Fall 7}

Die Untersuchung einer 21/2 Jahre alten Quarter-HorseStute fand wegen der Lahmheit statt, die sich in den letzten 10 Wochen des Trainings entwickelt hatte. Im Alter von 9 Monaten hatte man bei der Stute osteochondrale Fragmente am distalen mittleren Kamm der linken Tibia und am lateralen Rollkamm des linken Talus operativ entfernt. Die Stute ging hinten links lahm. Beide Metatarsophalangealgelenke waren deutlich vermehrt gefüllt, und das Beugen dieser Gelenke rief eine Schmerzreaktion hervor. Alle Fesselgelenke wurden röntgenologisch untersucht. Es fanden sich multiple rundliche Knochengebilde in beiden Metatarsophalangealgelenken. Der Sagittalkamm wies UnregelmäBigkeiten auf, und eine leichte Osteophytenbildung war vorhanden (Abb. 8). Knochendefekte von solcher Größe, daß sie als Ursprungsort der Knochenfragmente in den Gelenken in Frage gekommen wären, waren nicht zu entdekken. Es waren Unregelmäßigkeiten an der Dorsalfläche des Sagittalkamms am distalen Ende der beiden dritten Metakarpalknochen sichtbar. Im linken vorderen Fesselgelenk

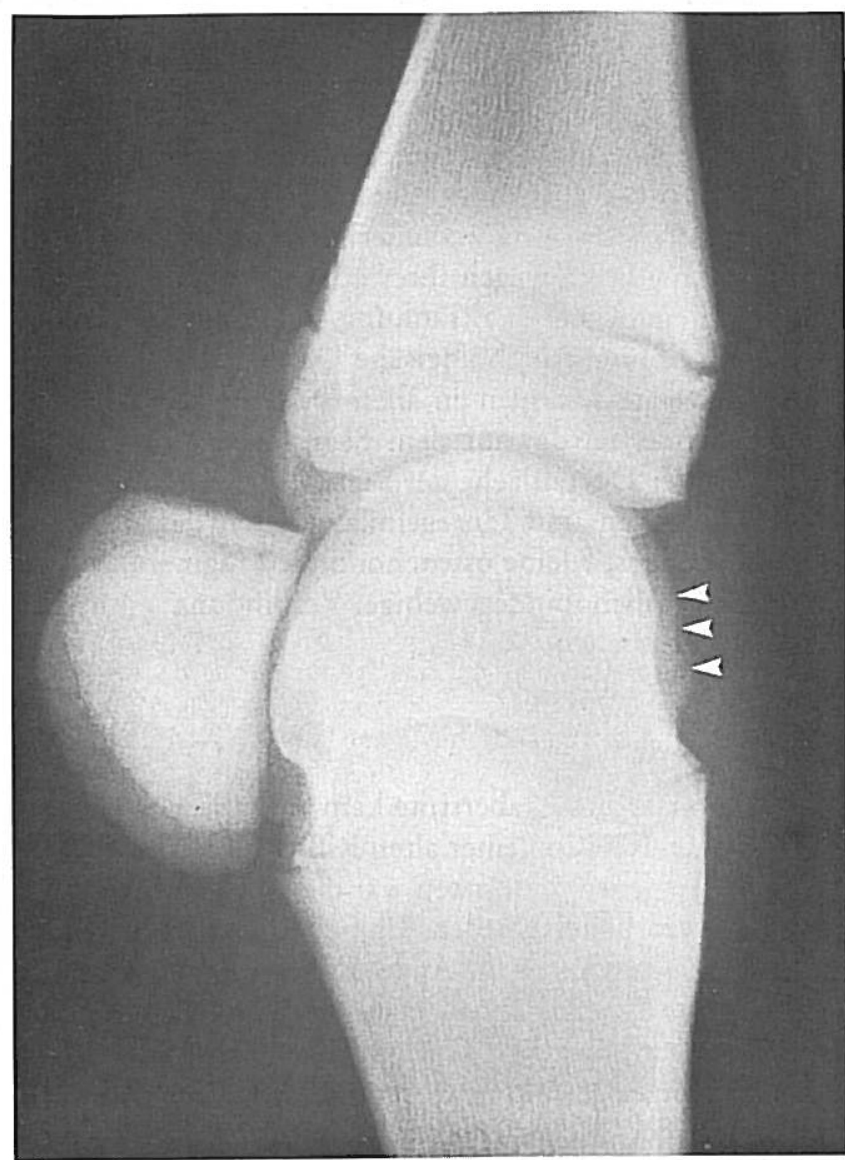

Abb. 6: Seitliche Röntgenaufnahme vom rechten Metakarpophalangealgelenk, Pferd 6 . Zu beachten die Unregelmäßigkeiten am Sagittalkamm (weiße Pfeile).

befand sich ein kleines Knochenfragment in diesem Bereich. Man aspirierte Synovialflüssigkeit aus beiden Metatarsophalangealgelenken und führte sie der physikalischen und zytologischen Analyse zu. Als einzige Abweichung war eine schwache Muzingerinselbildung in der Flüssigkeit aus dem rechten Metatarsophalangealgelenk festzustellen.

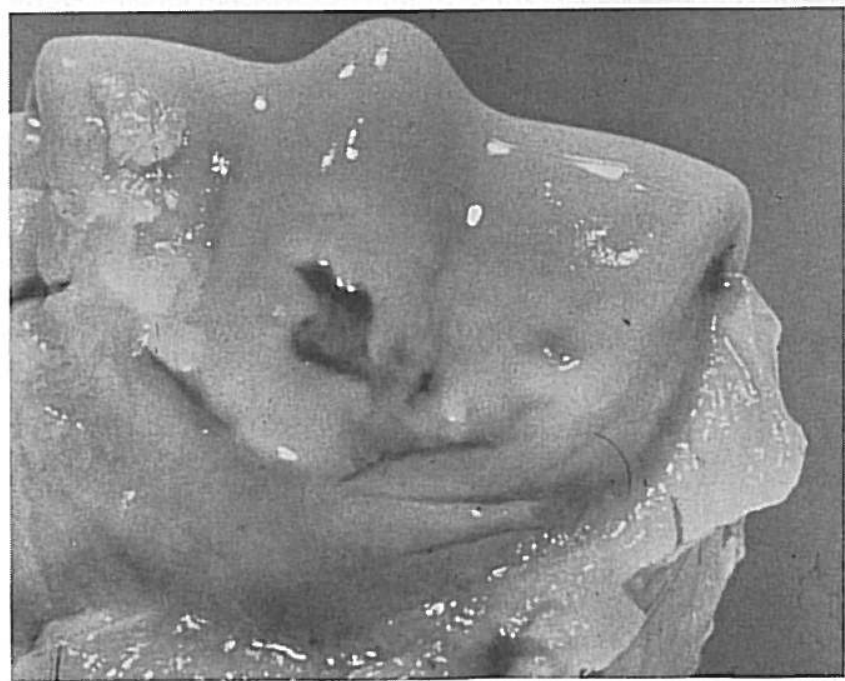

Abb. 7: Distalende des linken Os metatarsale tertium bei der Sektion, Pferd 6. Zu beachten die Knorpelerosion und der bloßgelegte subchondrale Knochen an der Dorsalfläche des Sagittalkamms. 
Mit Hilfe eines arthroskopischen Eingriffs wurden die Knochenfragmente aus den beiden hinteren Fesselgelenken entfernt. Die Metakarpophalangealgelenke operierte man nicht, da sie bei der Beugeprobe nicht schmerzhaft und die Röntgenbefunde von geringer Bedeutung waren. Im linken hinteren Fesselgelenk wurden 2 rundliche Knochenfragmente von der Dorsalfläche des Sagittalkamms entfernt, ebenso ein drittes Fragment, das sich lose im Gelenk gefunden hatte (Abb. 9). Im rechten Metatarsophalangealgelenk entfernte man ein großes Fragment von der Dorsolateralfläche und ein kleineres, das sich an der Dorsomedialfläche des Sagittalkamms befand. Die Knochenfragmente, die in beiden Fesselgelenken vom Sagittalkamm abgelöst worden waren, besaßen eine lose Bindegewebsverbindung zum subchondralen Knochen. Die postoperative Rekonvaleszenzphase verlief komplikationslos. Die histologische Untersuchung der entfernten Fragmente ergab, daß es sich um normales Bälkchenknochengewebe mit einer umgebenden Schicht hyalinen Knorpels (1 Fragment) oder Faserknorpels handelte (Abb. 10). Dies stimmte offenbar mit der Osteochondritis dissecans überein, bei der Knorpelfragmente einen eigenen Ossifikationskern entwickeln, aber mit der Gelenkfläche verbunden bleiben.

Die Stute erhielt nach der Operation 6 Monate Schonung mit Weidegang. Danach war keine Lahmheit mehr sichtbar, und die Metatarsophalangealgelenke waren nur noch zu einem sehr geringen Grad vermehrt gefüllt.

\section{Fall 8}

Eine 5 Jahre alte American-Saddlebred-Stute kam zur Lahmheitsuntersuchung. In den vorausgegangenen 2 Mo-

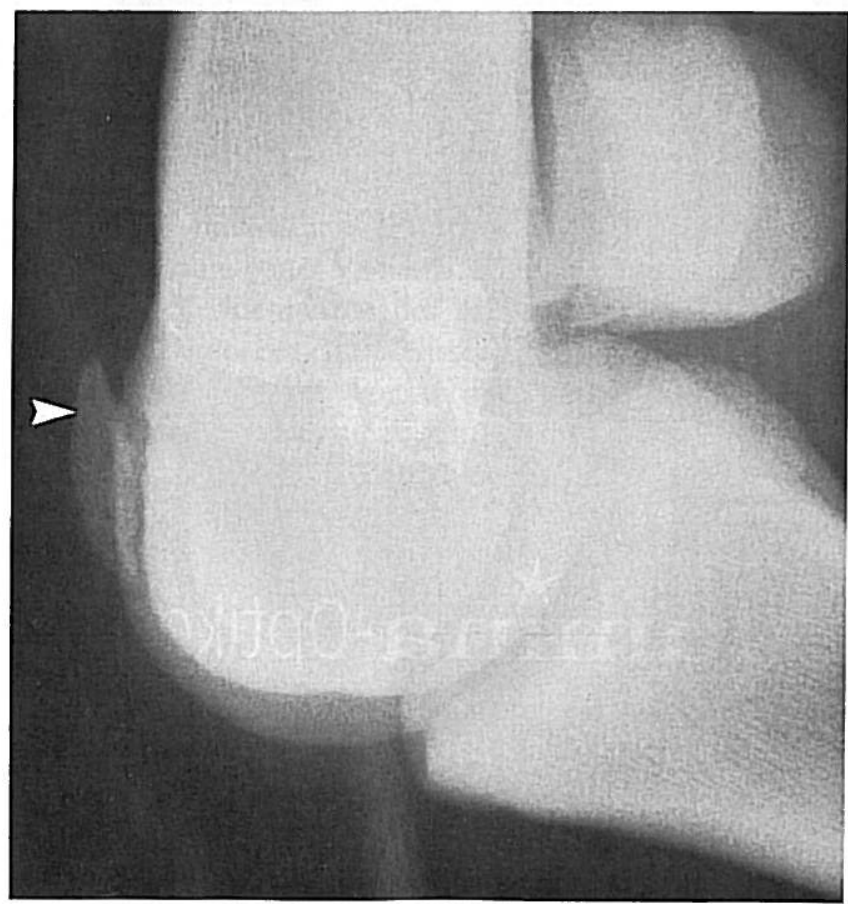

Abb. 8: Seitliche Röntgenaufnahme des linken Metatarsophalangealgelenks (gebeugt), Pferd 7. Das Knochenfragment (weißer Pfeil) befindet sich nahe bei einem Bereich von Unregelmäßigkeiten dorsal am Sagittalkamm. naten war die Stute zur Schonung auf der Weide gewesen. Alle Fesselgelenke waren sichtbar vermehrt gefüllt, besonders gut zu erkennen im Fall des linken Metatarsalgelenks. Im Trab war hinten links eine Lahmheit feststellbar, die sich nach der Fesselgelenksbeugeprobe noch verstärkte. Mit passivem Beugen des linken Metakarpophalangeal- und des linken Metatarsophalangealgelenks ließ sich deutlich Schmerz auslösen, nicht aber mit demjenigen der rechten Gliedmaßen.

Auf den Röntgenaufnahmen aller Fesselgelenke traten ähnliche Läsionen zutage. An der Dorsalfläche des Sagittalkamms waren Unregelmäßigkeiten und Knochendefekte vorhanden. Die proximalen Sesambeine des linken Vorderbeins wiesen eine periartikuläre Osteophytenbildung auf. Die Besitzer entschieden sich dafür, leichte Arbeit mit der Stute für Turnierzwecke zu beginnen und zur Besserung der Lahmheit eine Phenylbutazontherapie durchzuführen. 4 Monate später traf eine Benachrichtigung über den weiteren Verlauf ein, die besagte, daß das Pferd unter der Phenylbutazonapplikation an jedem zweiten Tag gute Leistungen erbracht habe.

\section{Diskussion}

In dieser Reihe von Fällen lautete bei 8 Pferden die Diagnose Osteochondritis dissecans des Sagittalkamms der Ossa metacarpalia und metatarsalia tertia. 7 Pferde waren $2^{1 / 2}$ Jahre alt oder jünger. Das stimmt mit dem allgemeinen Trend überein, daß die Osteochondritis dissecans in anderen Gelenken bei jüngeren Pferden festzustellen ist (Stromberg, 1976; Olsson et al., 1978). 6 der erkrankten Pferde wa-

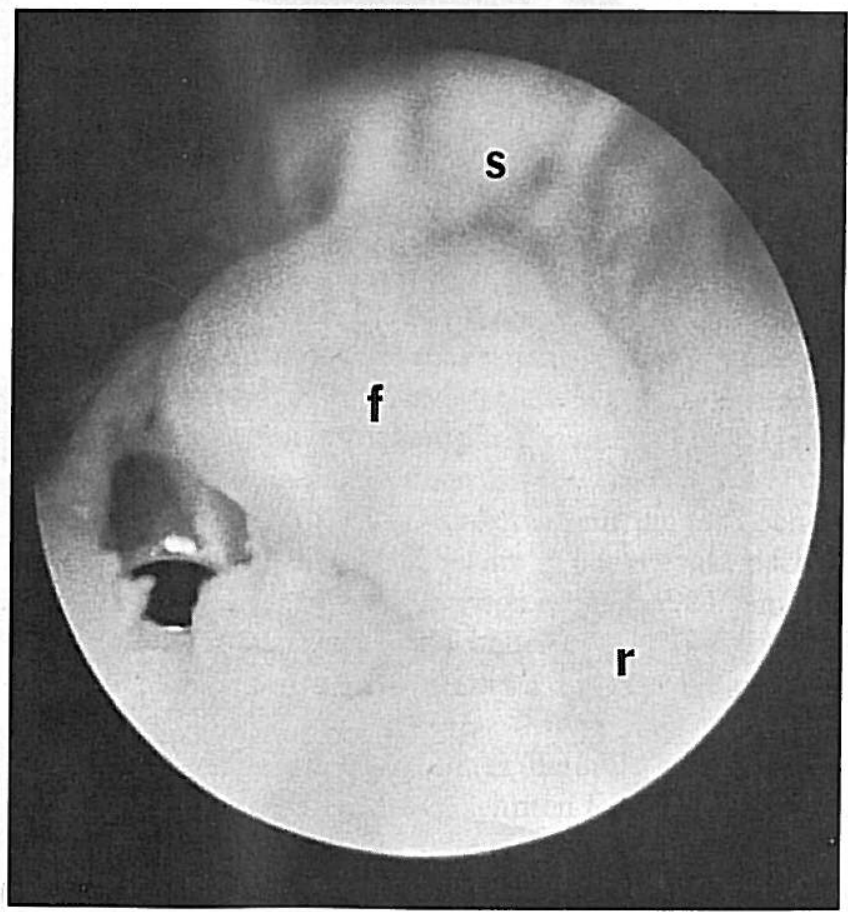

Abb. 9: Photographie, die während des arthroskopischen Eingriffs am linken Metatarsophalangealgelenk von Pferd 7 aufgenommen wurde. Ein Knochenfragment $(f)$ ist locker mit der Dorsalfiäche des Sagittalkamms (r) verbunden. $S$ = Tunica synovialis. Neben dem Knochenfragment ist die Abflußkanüle zu sehen. 
ren Stuten, was im Gegensatz zu Berichten steht, nach denen die Osteochondritis dissecans an anderen Stellen öfter bei Hengsten auftritt (Stromberg, 1976; Lindsell et al., 1983). Das Problem bei Pferden mit Osteochondritis dissecans am Sagittalkamm stellte sich entweder nur als vermehrte Füllung der Fesselgelenke (Nr. 1 und 3) oder als verstärkte Gelenkfüllung zusammen mit Lahmheit (Nr. 2, 4 und 8) dar. Bei 2 Pferden (Nr. 2 und 7) entwickelten sich die Erscheinungen während des Renntrainings. Von den übrigen dagegen wurde keines gearbeitet. 4 waren ein Jahr alt oder jünger und waren noch gar nicht in Arbeit genommen worden.

Im typischen Fall bestand die vermehrte Füllung an 2 oder mehr Fesselgelenken. Die Lahmheit war dabei an einem Bein besonders deutlich. Durch passives Beugen der erkrankten Fesselgelenke ließ sich jedoch in den meisten Fällen eine Schmerzreaktion auslösen und häufig die Lahmheit verstärken. Vermehrte Gelenkfüllung und Lahmheit sind gewöhnlich auch Anzeichen einer Osteochondrosis dissecans im Sprung- und Kniegelenk bei Pferden; unter Umständen sind in diesen Fällen aber Beugeproben erforderlich, um eine Lahmheit hervorzurufen (Lindsell et al., 1983); Pascoe et al., 1984; Fischer et al., 1984).

Die Röntgenuntersuchung ergab, daß alle Pferde Knochenläsionen am Sagittalkamm in mindestens 2 Fesselgelenken aufwiesen. Nur 1 Pferd (Nr. 7) zeigte klinische oder röntgenologische Erscheinungen von Osteochondrosis dissecans in einem anderen Gelenk. Röntgenbefunde am Sagittalkamm waren charakteristisch für die Osteochondrosis dissecans (Stromberg, 1976; Stromberg et al., 1978; Pappas, 1981). Die pathologischen Veränderungen rangierten von einer leichten Abflachung des Sagittalkamms (Nr. 1 und 2) bis zu ausgedehnten unregelmäßigen subchondralen Knochendefekten. Am deutlichsten traten sie an der Dorsalfläche des Sagittalkamms hervor. Bei 4 Pferden waren multiple Knochenfragmente sichtbar. Die Fragmente lagen entweder nahe bei der subchondralen Knochenaussparung (Nr. 1, 4 und 6) oder waren auch weiter davon entfernt (Nr. 7). Bei einem Pferd (Nr. 3) schwächte sich die Abflachung im Laufe von 9 Monaten etwas ab. Es liegen Berichte über eine Verkleinerung des subchondralen Knochendefekts bei Osteochondrosis dissecans der lateralen Femurtrochlea bei Pferden vor (Stromberg, 1976), aber dies scheint nicht als Regelfall zu gelten. Die Größe der verdichteten Knochengewebsstellen in der Nähe des Sagittalkamms nahm bei einem Pferd (Nr. 1) innerhalb von 2 Jahren zu.

Bei der Osteochondrosis dissecans von Pferden hängt das Alter, in dem die irritierende Wirkung der Knochenschädigungen zu klinischen Erscheinungen führt, hauptsächlich von der Schwere der Läsionen ab. Leicht betroffene Stellen bleiben unentdeckt, bis die erkrankten Pferde ins Training genommen werden (Stromberg, 1979). In der beschriebenen Reihe von Patienten bestand nicht in allen Fällen eine Beziehung zwischen dem Einsetzen klinischer Erscheinungen und dem Grad der röntgenologischen Veränderungen. Bei allen Pferden waren 2 oder mehr Fesselgelenke ähnlich stark betroffen, wie aus der Deutlichkeit der auf den Röntgenbildern sichtbaren Knochenläsionen hervorging. Im

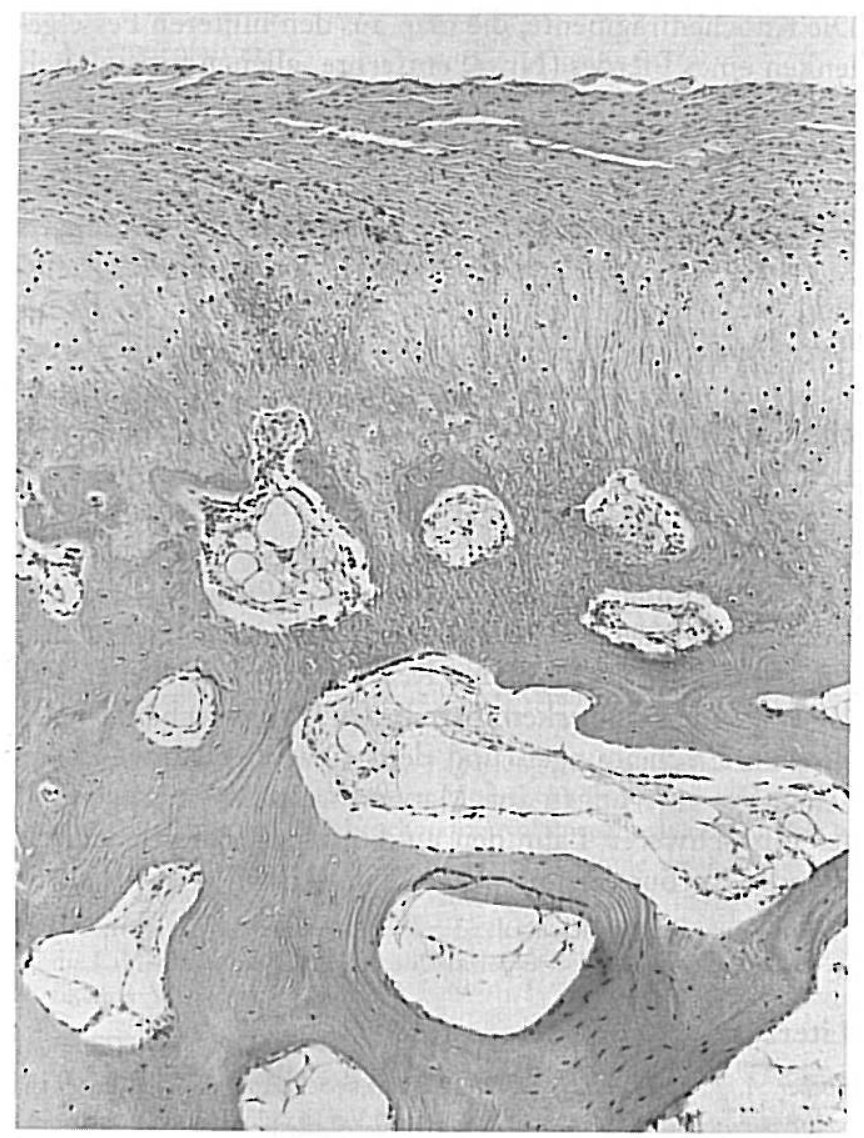

Abb. 10: Photomikrographie des Knochenfragments, das vom Sagittalkamm des linken Metatarsophalangealgelenks von Pferd 7 entfernt wurde. Zu beachten der Faserknorpel auf dem Bälkchenknochengewebe (H \& E stain; $\times 10$ ).

Lahmheitsgrad und in der Schmerzstärke, ausgelöst durch die Beugung des Fesselgelenks, bestanden aber beträchtliche Unterschiede zwischen den Gliedmaßen. Die beiden Pferde mit der höchstgradigen Lahmheit waren Fohlen, die nicht gearbeitet wurden (Nr. 4 und 6). Röntgenologisch besaßen diese beiden Fohlen multiple Knochenfragmente am Sagittalkamm. Man betrachtete diese Veränderungen als gravierender als diejenigen von Pferden mit wenig oder keinen Knochenfragmenten. Andererseits ging aber ein anderes Pferd (Nr. 7) mit Knochensplittern in allen Fesselgelenken erst'nach mehreren Rennen lahm.

Es mag wohl eine erbliche Disposition für die Osteochondrosis bei Pferden bestehen, aber sie ist nicht exakt nachgewiesen (Stromberg et al., 1978; Fischer et al., 1984). Interessant ist, daß sich bei der besprochenen Patientengruppe bei 2 Halbschwestern (Nr. 2 und 3) etwa dieselben Knochenläsionen am Sagittalkamm fanden.

Die makroskopisch-pathologischen Befunde bestanden bei 2 Pferden (Nr. 4 und 6) in ausgedehnten Knorpelerosionen, Riß- und Flapbildung sowie subchondralen Knochendefekten. Betroffen war davon der Sagittalkamm, aber nicht das übrige Fesselgelenk. Diese Befunde stehen in Übereinstimmung mit makroskopisch-pathologischen Beschreibungen von Osteochondrosis dissecans bei anderen Pferden (Stromberg, 1976; Rejno et al., 1978; Pascoe et al., 1984). 
Die Knochenfragmente, die man aus den hinteren Fesselgelenken eines Pferdes (Nr. 7) entfernte, glichen histologisch den osteochondralen Fragmenten anderer Pferde mit Osteochondrosis dissecans (Stromberg, 1976; Rejno et al., 1978). Um einen Kern aus Bälkchenknochen lag auf einer Seite normaler Gelenkknorpel und auf der anderen Faserknorpel. Am einen Ende der Splitter hingen Bindegewebsreste, wahrscheinlich dort, wo die Fragmente noch mit dem Defekt am Sagittalkamm verbunden gewesen waren. Bei Knochenschädigungen im Zusammenhang mit Osteochondrosis dissecans finden sich beim Pferd nicht selten freie Fragmente oder Gelenkskörper mit einem Inhalt aus Knochengewebe (Stromberg, 1976; Rejno et al., 1978; Olsson. et al., 1978).

Die Beurteilung von Behandlung und Prognose gestaltet sich schwierig wegen der geringen Zahl bisher untersuchter Pferde. Bei der vorliegenden Reihe von beschriebenen Fällen trat auch keine erkennbare Beziehung zwischen den klinischen Erscheinungen und dem Grad der röntgenologischen Veränderungen auf. Man euthanasierte 2 junge Pferde mit schwerer Lahmheit und multiplen Knochenfragmenten, verbunden mit dem Sagittalkamm (Nr. 4 und 6).

\section{Literatur}

Fischer, A. T., and Barclay, W. P. (1984): Osteochondrosis dissecans in the horse. Compend. Contin. Educ. Pract. Vet. 6, 123-131.

Lindsell, C. E., Hilbert, B. J., and McGill, C. A. (1983): A retrospective study of osteochondrosis dissecans in 21 horses. Aust. Vet. J. 60, 291-293.

McIlwraith, C. W. (1982): OCD and subchondral cystic lesions. Proc. Equine Orthop. 10th Ann. Vet. Surg. Forum, 48-50.

Olsson, S. E., and Reiland, S. (1978): The nature of osteochondrosis in animals. Summary and conclusions with comparative aspects on OCD in man. Acta Radiol. (Suppl.) (Stockh.) 358, 299-306.

Pappas, A. M. (1981): Osteochondrosis dissecans. Clin. Orthop. 158, 59-69.

Pascoe, J. R., Pool, R. R., Wheat, J. D., et al. (1984): Osteochondral defects of the lateral trochlear ridge of the distal femur of the horse. Clinical, radiographic, and pathological examination and results of surgical treatment. Vet. Surg. 13, 99-110.

Rejno, S., and Stromberg, B. (1978): Osteochondrosis in the horse II. Pathology. Acta Radiol. (Suppl.) (Stockh.) 358, 153-178.

Rooney, J. R. (1975): Osteochondrosis in the horse. Parts 1 and 2. Mod. Vet. Pract. $56(1), 41-43 ; 56(2), 112-116$.
Ein weiteres junges Pferd (Nr. 1), das nur sehr geringgradig lahm ging, aber Schmerzhaftigkeit bei der Fesselgelenksbeugung zeigte und Knochensplitter am Sagittalkamm aufwies, wurde durch Schonung schmerzfrei. Im einzigen noch behandelten Fall mit röntgenologisch sichtbaren Knochenfragmenten (Nr. 7) entfernte man die Bruchstükke. Zur Bewertung dieser Therapie bedarf es der chirurgischen Eingriffe an weiteren Pferden mit solchen Fragmenten. Bei anderen Patienten, die zur Entfernung osteochondraler Splitter aus den Fesselgelenken operiert wurden, haben wir mit dem Arthroskop relativ kleine Unregelmäßigkeiten am Sagittalkamm gesehen. Von den 4 Pferden mit Unebenheiten am Sagittalkamm oder Abflachung des Sagittalkamms (Nr. 2, 3, 5, 8) wurden 3 lediglich mit Schonung gesund (und eines ging nachher wieder erfolgreich Rennen). Mit einem wurde die Arbeit unter Phenylbutazontherapie ohne Schonphase nach der Erstellung der Diagnose direkt fortgesetzt.

Bei der Differentialdiagnose einer vom Fesselgelenk ausgehenden Lahmheit muß die Osteochondrose des Sagittalkamms der dritten Metakarpal- und Metatarsalknochen besonders bei jungen Pferden berücksichtigt werden.

Stromberg, B. (1976): Osteochondrosis dissecans of the stifle joint in the horse: a clinical, radiographic and pathologic study. J. Am. Vet. Radiol. Soc. XVII, 117-124.

Stromberg, B., and Rejno, S. (1978): Osteochondrosis in the horse. I. A. clinical and radiologic investigation of osteochondrosis dissecans in the knee and hock joint. Acta Radiol. (Suppl.) (Stockh.) 358, 139-152.

Stromberg, B. (1979): A review of the salient features of osteochondrosis in the horse. Equine Vet. J. 11, 211-214.

Joln V. Yovich, BSc, BVMS

Department of Clinical Sciences

College of Veterinary Medicine and Biomedical Sciences

Colorado State University

Fort Collins, CO 80523

USA

Erschienen im Journal of the American Veterinary Medical Association 186 (1985), 1186-1191. Übersetzt und veröffentlicht mit freundlicher Genehmigung der American Veterinary Medical Association 